\title{
La melatonina como un factor promotor de la diferenciación neuronal: implicaciones en el tratamiento de las demencias
}

\author{
Gloria Benítez-King, ${ }^{1}$ Marcela Valdés-Tovar, ${ }^{1}$ Vanessa Maya-Ampudia, ${ }^{1}$ Graciela Jiménez-Rubio, ${ }^{1}$ \\ Aline Domínguez-Alonso, ' Agustín Riquelme,' Tania Galván-Arrieta,' Héctor Solís-Chagoyán,' \\ Salvador Alarcón, ${ }^{1}$ Julia Moreno, ${ }^{1}$ Óscar Ugalde, ${ }^{2}$ Carlos Berlanga ${ }^{2}$
}

Artículo original

\section{SUMMARY}

Dementias are progressive and neurodegenerative neuropsychiatric disorders, with a high worldwide prevalence. These disorders affect memory and behavior, causing impairment in the performance of daily activities and general disability in the elders. Cognitive impairment in these patients is related to anatomical and structural alterations at cellular and sub-cellular levels in the Central Nervous System. In particular, amyloid plaques and neurofibrillar tangles have been defined as histopathological hallmarks of Alzheimer's disease. Likewise, oxidative stress and neuroinflammation are implicated in the etiology and progression of the disease.

Neuronal precursors from human olfactory neuroepithelium have been recently characterized as an experimental model to identify neuropsychiatric disease biomarkers. Moreover, this model not only allows the study of neuropsychiatric physiopathology, but also the process of neurodevelopment at cellular, molecular and pharmacological levels.

This review gathers the evidence to support the potential therapeutic use of melatonin for dementias, based on its antioxidant properties, its anti-inflammatory effect in the brain, and its ability to inhibit both tau hyper-phosphorylation and amyloid plaque formation. Furthermore, since melatonin stimulates neurogenesis, and promotes neuronal differentiation by inducing the early stages of neuritogenesis and dendrite formation, it has been suggested that melatonin could be useful to counteract the cognitive impairment in dementia patients.

Key words: Melatonin, dementia, biomarkers, neuroepithelium, neuritogenesis.

\section{RESUMEN}

Las demencias son enfermedades neuropsiquiátricas, progresivas, neurodegenerativas y con una alta prevalencia a nivel mundial. Ocupan uno de los primeros lugares como enfermedades que causan incapacidad en los adultos mayores. En estos pacientes el Sistema Nervioso Central presenta alteraciones anatómico-estructurales a nivel celular y subcelular que se asocian con deficiencias cognitivas. En particular, en la enfermedad de Alzheimer se han caracterizado marcadores histopatológicos como las placas amiloides y las marañas neurofibrilares. Se sabe que el estrés oxidativo y la neuroinflamación participan en la etiología y el desarrollo de la enfermedad. Recientemente se caracterizó a los precursores neuronales del neuroepitelio olfatorio humano como un modelo experimental adecuado para identificar biomarcadores de rasgo y para estudiar la fisiopatología de diversas enfermedades neuropsiquiátricas, así como el proceso del neurodesarrollo, a nivel celular, molecular y farmacológico. En este trabajo se presenta la evidencia que sustenta que la melatonina puede ser útil en el tratamiento de las demencias, por su capacidad antioxidante, por su efecto anti-inflamatorio, así como por el efecto inhibidor de la hiperfosforilación de la proteina tau y de la formación de placas amiloides. Además, al estimular la formación de nuevas neuronas, la neuritogénesis en sus etapas tempranas y la formación de dendritas, la melatonina podría contribuir a contrarrestar la pérdida de las funciones cognitivas que se observa en estos padecimientos.

Palabras clave: Melatonina, demencia, biomarcadores, neuroepitelio, neuritogénesis.
Las demencias son enfermedades neurodegenerativas que afectan, a nivel mundial, a cerca de 30 millones de personas. ${ }^{1,2}$ Ocupan el sexto lugar entre las enfermedades mentales que causan incapacidad y cada año se presentan 4.6 millones de casos nuevos. ${ }^{3}$ La enfermedad de Alzheimer (EA) es la forma más común de las demencias y afecta principalmente a los adultos mayores. Las tasas de prevalencia según la edad oscilan entre 5 y $8 \%$ en las personas que tienen arriba de 65 años, 15 a $20 \%$ en personas con una edad por arriba de los 75 años y 25 a $50 \%$ en personas con una edad mayor a los 85 años. ${ }^{4,5}$

Departamento de Neurofarmacología, Subdirección de Investigaciones Clínicas, Instituto Nacional de Psiquiatría Ramón de la Fuente Muñiz. Dirección de Servicios Clínicos. Instituto Nacional de Psiquiatría Ramón de la Fuente Muñiz.

Correspondencia: Dra. Gloria Benítez-King. Departamento de Neurofarmacología, Subdirección de Investigaciones Clínicas, Instituto Nacional de Psiquiatría Ramón de la Fuente Muñiz. Calz. México-Xochimilco 101, San Lorenzo Huipulco, Tlalpan, 14370 México DF. Tel. (52-55) 4160-5097. Fax.(52-55) 5513-3722. E-mail: bekin@imp.edu.mx 
Los pacientes con EA presentan alteraciones en la cognición (agnosia), en el ciclo sueño-vigilia y en la habilidad para realizar actividades cotidianas (apraxia), entre otros síntomas. ${ }^{4,6}$ En el Sistema Nervioso Central (SNC) se observan alteraciones anatómico-estructurales, celulares y subcelulares. ${ }^{7,8}$ Con técnicas de resonancia magnética transcraneal y tomografía de emisión de positrones se observa, a nivel macroscópico, atrofia cerebral generalizada ${ }^{9}$ y la pérdida gradual de la materia gris formada por núcleos neuronales y dendritas. ${ }^{10}$ La atrofia involucra al sistema límbico (hipocampo, amígdala y giro parahipocampal), a la corteza cerebral, a la corteza entorrinal, a la corteza de asociación y otras regiones subcorticales que incluyen el sistema colinérgico del prosencéfalo basal, el cuerpo estriado, el tálamo y el cerebelo, así como los lóbulos frontal, temporal, parietal y occipital. ${ }^{9,11}$ Las alteraciones en las regiones cerebrales están relacionadas con las deficiencias cognitivas que se presentan, tales como las afasias, que están causadas por el daño del área de Broca y/o de Wernicke en el hemisferio izquierdo y el fascículo arqueado que conecta ambas áreas; las apraxias, que están causadas por lesiones en el área de Broca y del cuerpo calloso, así como de las áreas de asociación parietal-frontal, la corteza motora y otras áreas asociadas al movimiento, según el tipo de apraxia. ${ }^{9}$ Además, los pacientes con EA tienen reducida la actividad neuronal en áreas de la corteza prefrontal y en todas las áreas de la formación hipocampal que incluyen al subiculum y que están asociadas con las deficiencias en la memoria episódica verbal. ${ }^{9}$

Una característica patológica que se presenta en las demencias es la disminución del volumen del hipocampo. ${ }^{11,12}$ Ésta se ha asociado con la pérdida neuronal en el hilus y en la región del cornus amonis CA1, entre otras. ${ }^{13,14}$ También se ha relacionado con la pérdida de axones y la disminución en el número de dendritas en la región CA1,15-17 así como con una reducción en las extensiones de las mismas, ${ }^{18}$ en el número de terminales dendríticas y en la longitud total de las dendritas en el giro parahipocampal. ${ }^{19}$ Esta disminución de la estructura de las dendritas reduce la cantidad de espinas y en consecuencia el ensamble de uniones sinápticas y de nuevos circuitos neuronales que participan en la plasticidad neuronal y la excitabilidad sináptica, inherentes a los procesos de memoria y aprendizaje.,11,15,20,21 En particular, en los pacientes con EA se ha observado la reducción de contactos sinápticos en la neocorteza y en la capa molecular del giro dentado del hipocampo, así como la pérdida de las aferencias provenientes de las neuronas localizadas en la corteza entorrinal. $^{22-24}$

\section{BIOMARCADORES DE LA DEMENCIA TIPO ALZHEIMER}

Aunados a los cambios estructurales a nivel cerebral, se han descrito varios marcadores biológicos de rasgo en el cere- bro de pacientes con el diagnóstico de EA. Entre éstos se encuentra el estrés oxidativo y se ha demostrado que los pacientes con EA tienen niveles elevados de radicales libres en la corteza frontal ${ }^{25}$ mediante técnicas de mapeo cerebral y resonancia magnética transcraneal. También los niveles de radicales libres se encuentran elevados en el plasma de estos pacientes y su capacidad antioxidante está disminuida. ${ }^{26}$ Se ha descrito, además, que el estrés oxidativo desempeña un papel crucial en la EA ya que produce una disminución en las densidades sinápticas, la reducción de factores neurotróficos como el NGF y el BDNF ${ }^{27}$ y causa el colapso del citoesqueleto neuronal ${ }^{28}$ que en su conjunto causa la atrofia cerebral generalizada. Asimismo, los radicales libres y las especies reactivas de oxígeno y nitrógeno (ROS/NOS) activan cascadas de señalización asociadas con la inflamación que, como se describe posteriormente, también tiene un papel importante en la etiología de los padecimientos neurodegenerativos.

Las placas amiloides difusas o neuríticas, también llamadas placas seniles, han sido consideradas como otro biomarcador de la EA. Se han descrito en la neocorteza y en el hipocampo durante las etapas tempranas de la enfermedad. ${ }^{29}$ Estas placas están formadas por la agregación de péptido beta amiloide $(A \beta)$ en la matriz extracelular del cerebro.

Las marañas neurofibrilares constituyen otro marcador histopatológico ${ }^{9,11}$ y se consideran uno de los principales criterios de diagnóstico post-mortem de la EA. Están formadas por agregados de filamentos helicoidales pareados (FHP) compuestos por la proteína tau hiperfosforilada. Ésta es una proteína asociada a los microtúbulos que se encuentran en los axones y que participan en el transporte axonal anterógrado. Al unirse a los microtúbulos, los estabiliza y actúa también promoviendo la polimerización de la tubulina. ${ }^{30} \mathrm{La}$ fosforilación excesiva de la tau causa la disociación de esta proteína de los microtúbulos y, en consecuencia, la despolimerización de la tubulina así como la pérdida de la asimetría morfofuncional de las neuronas, ${ }^{31}$ la reducción del número de contactos sinápticos y la interrupción del transporte axoplásmico. ${ }^{32}$

Actualmente se acepta que en la etiología de los padecimientos neurodegenerativos en general, y de la EA en particular, participa un componente de neuroinflamación crónica. En este sentido se ha demostrado que la microglia y los astrocitos activados se acumulan y se asocian a las placas seniles en el cerebro de estos pacientes, así como con una mayor cantidad de citocinas pro-inflamatorias, quimiocinas, proteínas del complemento, especies reactivas de oxígeno y nitrógeno y otros mediadores de inflamación. ${ }^{33,34}$ La activación de la microglia se produce por la presencia de los depósitos de $\mathrm{A} \beta$ y es, en principio, una respuesta protectora, ya que está dirigida a la eliminación de dichos depósitos por fagocitosis. ${ }^{35,36}$ En condiciones normales existe un equilibrio entre los procesos pro- y anti-inflamatorios que 
permite reparar el tejido y preservar la función neuronal. En los padecimientos neurodegenerativos este equilibrio se rompe y se desencadena un proceso crónico en el que se promueve el reclutamiento y la activación de la microglia y los astrocitos que producen diversos mediadores pro-inflamatorios y, a su vez, favorecen la activación de sistemas enzimáticos asociados a la inflamación. ${ }^{33}$ En respuesta al estrés celular, las neuronas, los astrocitos y el endotelio vascular generan más $\mathrm{A} \beta$, lo que perpetúa el proceso. ${ }^{33,37,38}$ La presencia de estos factores bioquímicos y celulares, aunada a la depleción de los mecanismos antioxidantes tisulares y a la pérdida de las funciones neuroprotectoras de los astrocitos, como la homeostasis del glutamato, ${ }^{39}$ contribuyen a generar un microambiente en donde se favorece la excitotoxicidad y la neurodegeneración, la retracción de las sinapsis con el consecuente déficit cognitivo, $\mathrm{y}$, eventualmente, la muerte neuronal. Por otra parte, en los últimos años se ha propuesto también que la inflamación sistémica juega un papel importante en la patogénesis de las demencias y se ha encontrado asociación entre los niveles elevados de marcadores inflamatorios periféricos como la proteína C-reactiva y la IL-6 séricas y un riesgo moderado de padecer demencia. ${ }^{40,41}$ A pesar de la gran cantidad de evidencia que existe en este tema, los mecanismos celulares y moleculares implicados en la neuroinflamación crónica asociada a las demencias son complejos y no han sido elucidados por completo. Asimismo, las estrategias terapéuticas que se han desarrollado en este ámbito, principalmente la administración de anti-inflamatorios no esteroideos (AINEs), han tenido resultados parciales en términos de prevención y de retraso en la progresión de la enfermedad, sin embargo no han sido eficaces en las etapas avanzadas de la misma. ${ }^{42}$

\section{MODELOS EXPERIMENTALES UTILIZADOS EN EL ESTUDIO DE LAS DEMENCIAS}

Los estudios para entender la fisiopatología de la EA se han realizado en diversos modelos animales y en cultivos celulares y organotípicos. ${ }^{43,44}$ Los estudios en roedores permitieron establecer que las lesiones en el hipocampo producidas por agentes químicos o mecánicos se asocian con deficiencias en la memoria espacial, ${ }^{45}$ en tanto que el modelo de cultivos organotípicos de esta estructura cerebral ha permitido definir las conexiones nerviosas y los núcleos neuronales involucrados en la memoria y la cognición así como abordar el estudio de fármacos neuroprotectores y neuroregeneradores potencialmente útiles para el tratamiento de la EA. ${ }^{46-48}$ Las rebanadas de hipocampo en cultivo permanecen viables por varias semanas, conservan su arquitectura tridimensional así como la integridad y funcionalidad de los circuitos neuronales ${ }^{49} \mathrm{y}$ en ellas se pueden evaluar topológicamente los efectos farmacológicos, tanto en neuronas adultas como en las nuevas neuronas que se forman en el giro dentado. ${ }^{47,50-52}$
En este modelo se han probado los efectos neurotóxicos del A $\beta$ sobre la agregación de la proteína tau y el efecto protector de la melatonina (MEL) sobre la neurotoxicidad inducida por el péptido A $325-35{ }^{44}$

Además de estos modelos experimentales, en años recientes se empezó a utilizar el neuroepitelio olfatorio (NEO) para estudiar posibles marcadores moleculares de la EA. ${ }^{53-55}$ Este epitelio contiene las neuronas más externas del organismo, por estar colocadas en una región periférica en contacto con el medio ambiente y por lo tanto accesibles para su obtención y aislamiento. ${ }^{56-58}$ Los primeros estudios de caracterización de biomarcadores se realizaron en biopsias del NEO obtenidas post-mortem. En una cohorte de 79 pacientes con diagnóstico de EA se describió un incremento en la cantidad de proteína tau-FHP, de agregados citoplasmáticos de $\mathrm{A} \beta$ y de alfa sinucleína como potenciales marcadores proteicos de la enfermedad.$^{59} \mathrm{El} \mathrm{NEO}$ es un tejido análogo al tubo neural que da origen al SNC y está constituido por varios tipos de células: las basales, las subtentaculares, las multipotenciales, los precursores neuronales y las neuronas sensoriales. ${ }^{60,61}$ Estas últimas se regeneran continuamente por la proliferación de las células multipotenciales que se diferencian para convertirse en neuronas sensoriales olfatorias. ${ }^{60,61}$ Recientemente desarrollamos un método para aislar las células del NEO de pacientes neuropsiquiátricos ambulatorios mediante la exfoliación no invasiva de la cavidad nasal y la implementación de las condiciones de cultivo para seleccionar, propagar, diferenciar y criopreservar a los precursores neuronales. ${ }^{62}$ Las células de linaje neuronal criopreservadas en bancos que posteriormente se descongelaron y se volvieron a cultivar, conservaron sus propiedades electrofisiológicas $\mathrm{y}$ fenotípicas ${ }^{62}$ y fueron capaces de desarrollar los arreglos del citoesqueleto característicos de la neuritogénesis. ${ }^{62}$ Estos datos sugieren que los precursores neuronales obtenidos de la cavidad nasal de los pacientes neuropsiquiátricos constituyen un modelo experimental adecuado para investigar la fisiopatología de la EA, los aspectos celulares y moleculares del neurodesarrollo tales como la diferenciación de las neuritas en axones y dendritas, así como diversas acciones farmacológicas sobre este proceso, y para la caracterización de biomarcadores de rasgo. ${ }^{62}$

\section{LA MELATONINA COMO UNA ALTERNATIVA EN EL TRATAMIENTO DE LAS DEMENCIAS}

En los últimos años se ha propuesto que la MEL (5-metoxyN-acetiltriptamina) puede ser útil en el tratamiento de la EA (para una revisión: Rosales et al., 2012). ${ }^{63}$ La administración de esta indolamina a sujetos que padecen EA causa una mejoría en los trastornos de los ritmos circadianos ${ }^{64-66}$ así como una reducción en la disfunción cognitiva. ${ }^{67}$ Los estudios preclínicos apoyan este concepto ya que la MEL actúa como un captador 
de radicales libres y por lo tanto reduce el estrés oxidativo y la apoptosis. ${ }^{68}$ En particular en las neuronas del hipocampo y en neuronas en cultivo la hormona disminuye los niveles de lipoperoxidación causados por la 1-metil-4-fenil-1,2,3,6-tetrahidropiridine (MPTP), ${ }^{69}$ la 6-hidroxidopamina (6-OHDA) ${ }^{70}$ y el ácido kaínico. ${ }^{71}$ También la MEL protege al citoesqueleto neuronal de la desorganización estructural que producen los radicales libres. ${ }^{28}$ Asimismo, en cultivos de microglia estimulados con el péptido A $\beta 1-42$, el pre-tratamiento con MEL reduce el ensamblaje y la actividad de la NADPH oxidasa y la consecuente producción de anión superóxido y ROS derivadas del mismo. ${ }^{72}$

El ácido ocadáico $(\mathrm{AO})$ aumenta el estrés oxidativo ${ }^{73} \mathrm{e}$ inhibe a las fosfatasas PP1 y PP2A, ${ }^{74}$ lo que incrementa la hiperfosforilación de la proteína tau, la formación de FHP y la retracción del citoesqueleto alrededor del núcleo. ${ }^{75} \mathrm{La}$ MEL, en concentraciones semejantes a las que circulan en el plasma y en el líquido cefalorraquídeo (1 y 100 nM, respectivamente) bloquea, previene y revierte el incremento en la lipoperoxidación y la apoptosis inducidas por el $\mathrm{AO}$ en las células N1E-115, así como el colapso del citoesqueleto, el que permanece organizado en el citoplasma y en las neuritas en presencia del AO y MEL. Resultados semejantes se obtuvieron cuando se probaron otros agentes oxidantes como el peróxido de hidrógeno y el haloperidol. 28,76,77 Por otro lado, la reducción de los niveles circulantes de MEL producida por la remoción quirúrgica de la glándula pineal (pinealectomía) se ha asociado con altos índices de estrés oxidativo, disminución de la memoria y desorganización del citoesqueleto en el hipocampo de la rata. ${ }^{78-81} \mathrm{El}$ tratamiento por una semana con MEL administrada intraperitonealmente revirtió estos efectos y los animales pinealectomizados e inyectados con MEL mostraron valores semejantes al grupo control en la cantidad de microtúbulos y microfilamentos determinados en la fracción citoesqueleto-membranal, ${ }^{81}$ lo que sugiere que el grado de estrés oxidativo, la organización del citoesqueleto y la cognición están relacionados.

\section{LA MELATONINA REDUCE LOS NIVELES DE TAU FOSFORILADA}

Otro de los cambios moleculares que bloquea la MEL es la hiperfosforilación de la proteína tau, que se presenta asociada al estrés oxidativo. En las células de neuroblastoma N1E-115 previamente incubadas con $50 \mathrm{nM}$ de AO, $100 \mu \mathrm{M}$ de peróxido de hidrógeno o $100 \mu \mathrm{M}$ de haloperidol, la MEL (1 o $100 \mathrm{nM}$ ) disminuye en un 100\% la cantidad relativa de tau fosforilada en la serina 404.77,82 Además, en modelos animales de EA, como los ratones 3xTg-AD, también se ha demostrado que la MEL disminuye los niveles de tau hiperfosforilada así como los síntomas conductuales similares a los de la demencia tales como la ansiedad y la pérdida de la conducta exploratoria. ${ }^{83}$ De manera análoga se ha descrito que en animales pinealectomizados hay un incremento en los niveles de tau hiperfosforilada asociados con un detrimento de la cognición. ${ }^{80}$

\section{LA MELATONINA ESTIMULA LA NEURITOGÉNESIS TEMPRANA Y LA DENDRITOGÉNESIS}

Además del efecto antioxidante, la MEL actúa como un modulador de la organización del citoesqueleto y, en consecuencia, del desarrollo de la polaridad morfofuncional en las neuronas. Este proceso implica la diferenciación de dos compartimentos celulares: el dominio axonal y el somatodendrítico. ${ }^{84}$ En cultivo, este proceso se inicia después de desprender a las neuronas del sustrato y de la resiembra. Las células redondas desarrollan una o múltiples neuritas que presentan conos de crecimiento en su extremo más distal. Posteriormente, una de las neuritas se alarga y se diferencia en el axón. Las neuritas cortas restantes se diferencian en dendritas y finalmente se lleva a cabo la polarización funcional y la formación de las sinapsis. ${ }^{84}$ Se ha demostrado que la calmodulina (CaM) cinasa II participa en la formación de las neuritas y de las dendritas mediante la fosforilación de las MAPs y de las STOPs (por sus siglas en inglés: "microtubule associated proteins" y "stable tubule only polypeptide", respectivamente) ${ }^{85,86}$ Las STOPs participan en el desarrollo y la diferenciación neuronal. Son proteínas que interaccionan con la CaM y se requieren en la formación de las neuritas. ${ }^{87}$ También estabilizan a los microtúbulos y se concentran en los dominios estables al frío y resistentes a fármacos en los axones maduros. ${ }^{88}$ En células en cultivo N1E-115, la MEL induce la formación de neuritas y su alargamiento mediante la estimulación de la polimerización de los microtúbulos y a través de un incremento en la organización de actina en los conos de crecimiento. ${ }^{89,90}$ Además, la indolamina estimula la neuritogénesis temprana mediante la activación de la proteína cinasa C (PKC) y de la RHO cinasa. ${ }^{90}$ Recientemente demostramos, en cultivos organotípicos de hipocampo, que la MEL incrementa la formación de dendritas así como su alargamiento y su complejidad, con un tiempo óptimo de seis horas en cultivo, en las neuronas e interneuronas de la zona del hilus que forma parte del circuito trisináptico del hipocampo y que tiene un papel clave en la integración de la memoria espacial. ${ }^{52,91,92}$ Estos efectos fueron dependientes de la concentración y se obtuvo una respuesta máxima con una concentración de $10^{-7} \mathrm{M}$ de MEL. En esta respuesta participa la CaM cinasa II ya que la formación de las dendritas no se estimula por MEL en presencia del inhibidor específico de esta enzima KN-62 y del inhibidor específico de la PKC, la bisindolilmaleimida (datos no publicados). Estos resultados y el hecho de que la indolamina en células en cultivo activa a la PKC e induce la fosforilación de la CaM y su traslado a la fracción citoesqueleto membranal, sugieren que esta enzima está vía abajo de la PKC en la vía de señalización de la MEL.93,94 


\section{LA MELATONINA TIENE EFECTOS ANTI-INFLAMATORIOS EN EL SISTEMA NERVIOSO CENTRAL}

Se sabe que la administración de MEL inhibe la activación de la microglia y la producción de citocinas pro-inflamatorias en modelos de neuroinflamación aguda causada por infección bacterian $\mathrm{a}^{95} \mathrm{o}$ por isquemia cerebral en ratas. ${ }^{96}$ Además, en el modelo de infección cerebral, el tratamiento con MEL $(100 \mathrm{mg} / \mathrm{kg})$ disminuye el número de neuronas apoptósicas, ${ }^{95}$ mientras que en el modelo de isquemia-reperfusión, la administración de MEL $(5 \mathrm{mg} / \mathrm{kg}$ ) reduce el infarto cerebral y las secuelas neuroconductuales asociadas. ${ }^{96}$ A nivel molecular, la MEL inhibe la expresión del ARNm de quimiocinas como CCL2 (MCP-1), CCL5 y CCL9 (MIP-1Y) inducidas por LPS en una línea celular de microglia. ${ }^{97}$ Este efecto fue mediado por la inhibición de la actividad transcripcional de NF-kB y STAT/GAS. ${ }^{97}$ En cultivos organotípicos de cerebro de ratón ${ }^{98}$ y de hipocampo de rata ${ }^{44}$ estimulados con los péptidos $A \beta 1-40$ y $A \beta 25-35$, respectivamente, la MEL disminuye la secreción de citocinas pro-inflamatorias y previene la activación de la microglia y de los astrocitos que se inducen por la exposición al péptido A $\beta 25-35 .{ }^{44}$ Aunado a estos efectos la MEL inhibe la expresión de las enzimas pro-inflamatorias iNOS y COX-2, en la línea celular de astrocitos C6, ${ }^{99}$ sin inhibir la enzima COX-1, por lo que se ha sugerido que la indolamina podría tener efectos terapéuticos similares a los de los AINEs, pero sin sus efectos adversos. ${ }^{100}$ La reducción en los niveles de citocinas pro-inflamatorias como TNF- $\alpha$ e IL-6, enzimas como iNOS y COX-2, y factores de transcripción como NF-кB, y la estimulación simultánea de sistemas antioxidantes, como los asociados a la cascada de Nrf2, entre otros, se consideran parte de los mecanismos moduladores de la melatonina en la neuroinflamación. ${ }^{101,102}$

La evidencia que se tiene a la fecha indica que la MEL puede ser útil en el tratamiento de la EA y de las taupatías en general, por su capacidad antioxidante, por su efecto sincronizador del ritmo sueño-vigilia, por su efecto anti-inflamatorio, así como por el efecto inhibidor de la hiperfosforilación de la tau y de la formación de placas amiloideas. En años recientes se demostró que la MEL estimula la formación de nuevas neuronas, la neuritogénesis en sus etapas tempranas, así como la formación de dendritas. Sin embargo no se sabe si la indolamina estimula la diferenciación morfofuncional en otras etapas de este proceso como la formación de axones, de espinas dendríticas, de sinapsis competentes y eléctricamente activas. Por todo lo señalado hasta ahora es de suma importancia continuar con el estudio del desarrollo neuronal en presencia de la MEL, definir los mecanismos de acción involucrados en este proceso, así como los efectos que tiene esta indolamina en los principales biomarcadores de rasgo de las demencias en los modelos de neurodegeneración establecidos, así como en los precursores neuronales obtenidos del neuroepitelio nasal de pacientes neuropsiquiátricos.

\section{REFERENCIAS}

1. Secretaría de Salud (SSA). México Sano. 2008; enero;1(1):11; http://portal.salud.gob.mx/descargas/pdf/period_mexsano/mexicosano_ene08. pdf, 26 de noviembre de 2012.

2. Llibre Rodríguez JJ, Ferri CP, Acosta D, Guerra M et al. Prevalence of dementia in Latin America, India, and China: a population-based cross-sectional survey. Lancet 2008;372(9637):464-474.

3. Baldereschi M, Di Carlo A, Amaducci L. Epidemiology of dementias. Drugs Today 1998;34(9):747-758.

4. American Psychiatric Association. Delirium, dementia, and amnestic and other cognitive disorders. En: First M (ed). Diagnostic and Statistical Manual of Mental Disorders. Cuarta edición. DSM-IV-TR®. Arlington, VA: American Psychiatric Publishing; 2000.

5. Rabins PV, Blacker D, Rovner BW, Rummans T Et al. American Psychiatric Association practice guideline for the treatment of patients with Alzheimer's disease and other dementias. Second edition. Am J Psychiatry 2007;164(12Supl):5-56.

6. Burns A, Byrne EJ, Maurer K. Alzheimer's disease. Lancet 2002;360(9327):163-165.

7. Pasantes H, Arias C, Massieu L, Zentella A et al. Enfermedades neurodegenerativas: mecanismos celulares y moleculares. México: Fondo de Cultura Económica; 1999.

8. Williams DR. Tauopathies: classification and clinical update on neurodegenerative diseases associated with microtubule-associated protein tau. Intern Med J Australia 2006;36(10):652-660.

9. Andersen P, Morris R, Amaral D, Bliss T, O'Keefe J. The Hippocampus Book. EUA: Oxford University Press; 2006.

10. Thompson PM, Hayashi KM, de Zubicaray G, Janke AL et al. Dynamics of gray matter loss in Alzheimer's disease. J Neurosci 2003;23(3):994-1005.

11. Squire L, Bloom FE, Spitzer NC, Squire LR et al. Fundamental Neuroscience. USA: Academic Press; 2008.

12. Caserta MT, Bannon Y, Fernandez F, Giunta B et al. Chapter 1 normal brain aging: Clinical, immunological, neuropsychological, and neuroimaging features. En: Minagar A (ed). International review of neurobiology, neurobiology of dementia. USA: Academic Press; 2009.

13. West MJ, Kawas CH, Stewart WF, Rudow GL et al. Hippocampal neurons in pre-clinical Alzheimer's disease. Neurobiol Aging 2004;25(9):1205-1212.

14. Zarow C, Vinters HV, Ellis WG, Weiner MW et al. Correlates of hippocampal neuron number in Alzheimer's disease and ischemic vascular dementia. Ann Neurol 2005;57(6):896-903.

15. Stuart G, Spruston N, Hausser M. Dendrites. USA: Oxford University Press; 2007.

16. Xekardaki A, Giannakopoulos $P$, Haller S. White matter changes in bipolar disorder, Alzheimer disease, and mild cognitive impairment: New insights from DTI. J Aging Res 2011;2011:286564.

17. Jawhar S, Trawicka A, Jenneckens C, Bayer TA et al. Motor deficits, neuron loss, and reduced anxiety coinciding with axonal degeneration and intraneuronal Abeta aggregation in the 5XFAD mouse model of Alzheimer's disease. Neurobiol Aging 2012;33(1):196 e29-40.

18. Yamada M, Wada Y, Tsukagoshi H, Otomo E et al. A quantitative Golgi study of basal dendrites of hippocampal CA1 pyramidal cells in senile dementia of Alzheimer type. J Neurol Neurosurg Psychiatry 1988;51(8):1088-1090.

19. Buell SJ, Coleman PD. Dendritic growth in the aged human brain and failure of growth in senile dementia. Science 1979;206(4420):854-856.

20. Samuel W, Masliah E, Hill LR, Butters N et al. Hippocampal connectivity and Alzheimer's dementia: effects of synapse loss and tangle frequency in a two-component model. Neurology 1994;44(11):2081-2088.

21. Tiraboschi P, Hansen LA, Alford M, Masliah E et al. Corey-Bloom J. The decline in synapses and cholinergic activity is asynchronous in Alzheimer's disease. Neurology 2000;55(9):1278-1283.

22. Hamos JE, DeGennaro LJ, Drachman DA. Synaptic loss in Alzheimer's disease and other dementias. Neurology 1989;39(3):355-361. 
23. Lassmann H, Fischer P, Jellinger K. Synaptic pathology of Alzheimer's disease. Ann N Y Acad Sci 1993;695:59-64.

24. Scheff SW, Price DA, Schmitt FA, Mufson EJ. Hippocampal synaptic loss in early Alzheimer's disease and mild cognitive impairment. Neurobiol Aging 2006;27(10):1372-1384.

25. Mandal PK, Tripathi M, Sugunan S. Brain oxidative stress: detection and mapping of anti-oxidant marker 'Glutathione' in different brain regions of healthy male/female, $\mathrm{MCI}$ and Alzheimer patients using non-invasive magnetic resonance spectroscopy. Biochem Biophys Res Commun 2012;417(1):43-48.

26. Repetto MG, Reides CG, Evelson P, Kohan $S$ et al. Peripheral markers of oxidative stress in probable Alzheimer patients. Eur J Clin Invest 1999;29(7):643-649.

27. Kermer P, Liman J, Weishaupt JH, Bahr M. Neuronal apoptosis in neurodegenerative diseases: from basic research to clinical application. Neurodegener Dis 2004;1(1):9-19.

28. Benítez-King G, Ortiz-López L, Jiménez G. Melatonin precludes cytoskeletal collapse caused by hydrogen peroxide: participation of protein kinase C. Therapy 2005;2(5):767-778.

29. Selkoe DJ. Toward a comprehensive theory for Alzheimer's disease. Hypothesis: Alzheimer's disease is caused by the cerebral accumulation and cytotoxicity of amyloid beta-protein. Ann N Y Acad Sci 2000;924:17-25.

30. Brandt R, Lee G. Orientation, assembly, and stability of microtubule bundles induced by a fragment of tau protein. Cell Motil Cytoskeleton 1994;28(2):143-154.

31. Alonso AD, Grundke-Iqbal I, Barra HS, Iqbal K. Abnormal phosphorylation of tau and the mechanism of Alzheimer neurofibrillary degeneration: sequestration of microtubule-associated proteins 1 and 2 and the disassembly of microtubules by the abnormal tau. Proc Natl Acad Sci USA 1997;94(1):298-303.

32. Griffin JW, Watson DF. Axonal transport in neurological disease. Ann Neurol 1988;23(1):3-13.

33. Rubio-Perez JM, Morillas-Ruiz JM. A review: inflammatory process in Alzheimer's disease, role of cytokines. Scientific World J 2012;2012:756357.

34. Agostinho P, Cunha RA, Oliveira C. Neuroinflammation, oxidative stress and the pathogenesis of Alzheimer's disease. Curr Pharm Des 2010;16(25):2766-2778.

35. Azizi G, Mirshafiey A. The potential role of proinflammatory and antiinflammatory cytokines in Alzheimer disease pathogenesis. Immunopharmacol Immunotoxicol 2012; DOI: 10.3109/08923973.2012.705292, ISSN: 1532-2513 (Electronic).

36. Schlachetzki JC, Hull M. Microglial activation in Alzheimer's disease. Curr Alzheimer Res 2009;6(6):554-563.

37. Rossner S, Lange-Dohna C, Zeitschel U, Perez-Polo JR. Alzheimer's disease beta-secretase BACE1 is not a neuron-specific enzyme. J Neurochem 2005;92(2):226-234.

38. Bulbarelli A, Lonati E, Brambilla A, Orlando A et al. Abeta42 production in brain capillary endothelial cells after oxygen and glucose deprivation. Mol Cell Neurosci 2012;49(4):415-422.

39. Rodriguez JJ, Olabarria M, Chvatal A, Verkhratsky A. Astroglia in dementia and Alzheimer's disease. Cell Death Differ 2009;16(3):378385.

40. Koyama A, O'Brien J, Weuve J, Blacker D, Metti AL, Yaffe K. The Role of Peripheral Inflammatory Markers in Dementia and Alzheimer's Disease: A Meta-Analysis. J Gerontol A Biol Sci Med Sci. 2012. DOI: 10.1093/gerona/gls187, ISSN: 1758-535X (Electronic).

41. Holmes C. Systemic inflammation and Alzheimer's Disease. Neuropathol Appl Neurobiol 2012. DOI: 10.1111/j.1365-2990.2012.01307.x, ISSN: 1365-2990 (Electronic).

42. Hoozemans JJ, Veerhuis R, Rozemuller JM, Eikelenboom P. Soothing the inflamed brain: effect of non-steroidal anti-inflammatory drugs on Alzheimer's disease pathology. CNS Neurol Disord Drug Targets 2011;10(1):57-67.
43. Lewis TL, Cao D, Lu H, Mans RA, Su YR et al. Overexpression of human apolipoprotein A-I preserves cognitive function and attenuates neuroinflammation and cerebral amyloid angiopathy in a mouse model of Alzheimer disease. J Biol Chem 2010;285(47):36958-968.

44. Hoppe JB, Frozza RL, Horn AP, Comiran RA et al. Amyloid-beta neurotoxicity in organotypic culture is attenuated by melatonin: involvement of GSK-3beta, tau and neuroinflammation. J Pineal Res 2010;48(3):230-238.

45. He FQ, Qiu BY, Zhang XH, Li TK et al. Tetrandrine attenuates spatial memory impairment and hippocampal neuroinflammation via inhibiting NF-kappaB activation in a rat model of Alzheimer's disease induced by amyloid-beta(1-42). Brain Res 2011;1384:89-96.

46. Garcia-Chavez D, Gonzalez-Burgos I, Letechipia-Vallejo G, Lopez-Loeza $\mathrm{E}$ et al. Long-term evaluation of cytoarchitectonic characteristics of prefrontal cortex pyramidal neurons, following global cerebral ischemia and neuroprotective melatonin treatment, in rats. Neurosci Lett. 2008;448(1):148-152.

47. Noraberg J, Poulsen FR, Blaabjerg M, Kristensen BW et al. Organotypic hippocampal slice cultures for studies of brain damage, neuroprotection and neurorepair. Curr Drug Targets CNS Neurol Disord 2005;4(4):435-152.

48. Cho S, Wood A, Bowlby MR. Brain slices as models for neurodegenerative disease and screening platforms to identify novel therapeutics. Curr Neuropharmacol 2007;5(1):19-33.

49. Gogolla N, Galimberti I, DePaola V, Caroni P. Staining protocol for organotypic hippocampal slice cultures. Nat Protoc 2006;1(5):2452-2456.

50. Benítez-King G, Domínguez-Alonso A, Ramírez-Rodríguez G. Neurocytoskeletal protective effect of melatonin: importance for morpho-functional neuronal polarization. Open Neuroendocrinology J 2010;3:105-111.

51. Ramírez-Rodríguez G, Ortiz-López L, Domínguez-Alonso A, BenitezKing GA et al. Chronic treatment with melatonin stimulates dendrite maturation and complexity in adult hippocampal neurogenesis of mice. J Pineal Res 2011;50(1):29-37.

52. Domínguez-Alonso A, Ramírez-Rodríguez G, Benítez-King G. Melatonin increases dendritogenesis in the hilus of hippocampal organotypic cultures. J Pineal Res 2012;52(4):427-436.

53. Tabaton M, Cammarata S, Mancardi GL, Cordone G et al. Abnormal taureactive filaments in olfactory mucosa in biopsy specimens of patients with probable Alzheimer's disease. Neurology 1991;41(3):391-394.

54. Talamo BR, Rudel R, Kosik KS, Lee VM et al. Pathological changes in olfactory neurons in patients with Alzheimer's disease. Nature 1989;337(6209):736-739.

55. Lee JH, Goedert M, Hill WD, Lee VM et al. Tau proteins are abnormally expressed in olfactory epithelium of Alzheimer patients and developmentally regulated in human fetal spinal cord. Exp Neurol 1993;121(1):93-105.

56. Jafek B, Johnson E, Eller P, Murrow B. Olfactory mucosal biopsy and related histology. En: Seiden A (ed). Taste and Smell Disorders. New York, NY: Thieme; 1997.

57. Lane AP, Gomez G, Dankulich $\mathrm{T}$, Wang $\mathrm{H}$ et al. The superior turbinate as a source of functional human olfactory receptor neurons. Laryngoscope 2002;112(7 Pt1):1183-1189.

58. Lanza DC, Deems DA, Doty RL, Moran D et al. The effect of human olfactory biopsy on olfaction: a preliminary report. Laryngoscope 1994;104(7):837-840.

59. Arnold SE, Lee EB, Moberg PJ, Stutzbach L et al. Olfactory epithelium amyloid-beta and paired helical filament-tau pathology in Alzheimer disease. Ann Neurol 2010;67(4):462-469.

60. Graziadei P, Monti-Graziadei G. The olfactory system: a model for the study of neurogenesis and axon regeneration in mammals. En: Cotman C (ed). Neuronal plasticity. New York: Raven Press; 1978.

61. Hahn CG, Han LY, Rawson NE, Mirza $\mathrm{N}$ et al. In vivo and in vitro neurogenesis in human olfactory epithelium. J Comp Neurol 2005;483(2):154-163. 
62. Benítez-King G, Riquelme A, Ortiz-López L, Berlanga C et al. A noninvasive method to isolate the neuronal linage from the nasal epithelium from schizophrenic and bipolar diseases. J Neurosci Methods 2011;201(1):35-45.

63. Rosales-Corral SA, Acuna-Castroviejo D, Coto-Montes A, Boga JA et al. Alzheimer's disease: pathological mechanisms and the beneficial role of melatonin. J Pineal Res 2012;52(2):167-202.

64. Cardinali DP, Furio AM, Brusco LI. Clinical aspects of melatonin intervention in Alzheimer's disease progression. Curr Neuropharmacol 2010;8(3):218-227.

65. Brusco LI, Marquez M, Cardinali DP. Monozygotic twins with Alzheimer's disease treated with melatonin: Case report. J Pineal Res 1998;25(4):260-263.

66. Jean-Louis G, Zizi F, von Gizycki H, Taub H. Effects of melatonin in two individuals with Alzheimer's disease. Percept Mot Skills 1998;87(1):331-339.

67. Brusco LI, Marquez M, Cardinali DP. Melatonin treatment stabilizes chronobiologic and cognitive symptoms in Alzheimer's disease. Neuro Endocrinol Lett 2000;21(1):39-42.

68. Reiter RJ. Oxidative damage in the central nervous system: protection by melatonin. Prog Neurobiol 1998;56(3):359-384.

69. Acuña-Castroviejo D, Coto-Montes A, Gaia Monti M, Ortiz GG et al. Melatonin is protective against MPTP-induced striatal and hippocampal lesions. Life Sci 1997;60(2):PL23-29.

70. Mayo JC, Sainz RM, Uria H, Antolin I et al. Inhibition of cell proliferation: a mechanism likely to mediate the prevention of neuronal cell death by melatonin. J Pineal Res 1998;25(1):12-18.

71. Tan DX, Manchester LC, Reiter RJ, Qi W et al. Melatonin protects hippocampal neurons in vivo against kainic acid-induced damage in mice. J Neurosci Res 1998;54(3):382-389.

72. Zhou J, Zhang S, Zhao X, Wei T. Melatonin impairs NADPH oxidase assembly and decreases superoxide anion production in microglia exposed to amyloid-beta1-42. J Pineal Res 2008;45(2):157-165.

73. Arendt T, Holzer M, Bruckner MK, Janke $C$ et al. The use of okadaic acid in vivo and the induction of molecular changes typical for Alzheimer's disease. Neuroscience 1998;85(4):1337-1340.

74. Bialojan C, Takai A. Inhibitory effect of a marine-sponge toxin, okadaic acid, on protein phosphatases. Specificity and kinetics. Biochem J 1988;256(1):283-290.

75. Lee J, Hong $\mathrm{H}, \mathrm{Im} \mathrm{J}$, Byun $\mathrm{H}$ et al. The formation of PHF-1 and SMI31 positive dystrophic neurites in rat hippocampus following acute injection of okadaic acid. Neurosci Lett 2000;282(1-2):49-52.

76. Jiménez-Rubio G, Benítez-King G, Ortiz-López L. Melatonin elicits neuritogenesis and reverses tau hyperphosphorylation in N1E-115 neuroblastoma cells treated with okadaic acid. En: Fernández A (ed). Focus on neuroblastoma research. Hauppauge, NY: Nova Science Publisher; 2007.

77. Benítez-King G, Ortiz-López L, Jiménez-Rubio G, Ramírez-Rodríguez G. Haloperidol causes cytoskeletal collapse in N1E-115 cells through tau hyperphosphorylation induced by oxidative stress: Implications for neurodevelopment. Eur J Pharmacol 2010;644(1-3):24-31.

78. Reiter RJ, Tan D, Kim SJ, Manchester LC et al. Augmentation of indices of oxidative damage in life-long melatonin-deficient rats. Mech Ageing Dev 1999;110(3):157-173.

79. Galano A, Tan DX, Reiter RJ. Melatonin as a natural ally against oxidative stress: a physicochemical examination. J Pineal Res 2011;51(1):1-16.

80. Ling $Z Q$, Tian $Q$, Wang $L, F u ~ Z Q$ et al. Constant illumination induces Alzheimer-like damages with endoplasmic reticulum involvement and the protection of melatonin. J Alzheimers Dis 2009;16(2):287-300.

81. Jiménez-Rubio G, Ortiz-López L, Benítez-King G. Melatonin modulates cytoskeletal organization in the rat brain hippocampus. Neurosci Lett 2012;511(1):47-51.

82. Benítez-King G, Tunez I, Bellon A, Ortiz GG et al. Melatonin prevents cytoskeletal alterations and oxidative stress induced by okadaic acid in N1E-115 cells. Exp Neurol 2003;182(1):151-159.
83. García-Mesa Y, López-Ramos JC, Gimenez-Llort L, Revilla $S$ et al. Physical exercise protects against Alzheimer's disease in 3xTg-AD mice. J Alzheimers Dis 2011;24(3):421-454.

84. Tahirovic S, Bradke F. Neuronal polarity. Cold Spring Harb Perspect Biol 2009;1(3):a001644.

85. Vaillant AR, Zanassi P, Walsh GS, Aumont A et al. Signaling mechanisms underlying reversible, activity-dependent dendrite formation. Neuron 2002;34(6):985-998.

86. Baratier J, Peris L, Brocard J, Gory-Faure S et al. Phosphorylation of microtubule-associated protein STOP by calmodulin kinase II. J Biol Chem 2006;281(28):19561-19569.

87. Guillaud L, Bosc C, Fourest-Lieuvin A, Denarier E et al. STOP proteins are responsible for the high degree of microtubule stabilization observed in neuronal cells. J Cell Biol 1998;142(1):167-179.

88. Slaughter T, Black MM. STOP (stable-tubule-only-polypeptide) is preferentially associated with the stable domain of axonal microtubules. J Neurocytol 2003;32(4):399-413.

89. Huerto-Delgadillo L, Antón-Tay F, Benítez-King G. Effects of melatonin on microtubule assembly depend on hormone concentration: role of melatonin as a calmodulin antagonist. J Pineal Res 1994;17(2):55-62.

90. Bellon A, Ortiz-López L, Ramírez-Rodríguez G, Antón-Tay F et al. Melatonin induces neuritogenesis at early stages in N1E-115 cells through actin rearrangements via activation of protein kinase $\mathrm{C}$ and Rho-associated kinase. J Pineal Res 2007;42(3):214-221.

91. Gilbert PE, Brushfield AM. The role of the CA3 hippocampal subregion in spatial memory: a process oriented behavioral assessment. Prog Neuropsychopharmacol Biol Psychiatry 2009;33(5):774-781.

92. Xavier GF, Costa VC. Dentate gyrus and spatial behaviour. Prog Neuropsychopharmacol Biol Psychiatry 2009;33(5):762-773.

93. Benítez-King G, Huerto-Delgadillo L, Antón-Tay F. Melatonin modifies calmodulin cell levels in MDCK and N1E-115 cell lines and inhibits phosphodiesterase activity in vitro. Brain Res 1991;557(1-2):289-292.

94. Soto-Vega E, Meza I, Ramírez-Rodríguez G, Benítez-King G. Melatonin stimulates calmodulin phosphorylation by protein kinase C. J Pineal Res 2004;37(2):98-106.

95. Wu UI, Mai FD, Sheu JN, Chen LY et al. Melatonin inhibits microglial activation, reduces pro-inflammatory cytokine levels, and rescues hippocampal neurons of adult rats with acute Klebsiella pneumoniae meningitis. J Pineal Res 2011;50(2):159-170.

96. Lee MY, Kuan YH, Chen HY, Chen TY et al. Intravenous administration of melatonin reduces the intracerebral cellular inflammatory response following transient focal cerebral ischemia in rats. J Pineal Res 2007;42(3):297-309.

97. Min KJ, Jang JH, Kwon TK. Inhibitory effects of melatonin on the lipopolysaccharide-induced CC chemokine expression in BV2 murine microglial cells are mediated by suppression of Akt-induced NF-kappaB and STAT/GAS activity. J Pineal Res 2012;52(3):296-304.

98. Clapp-Lilly KL, Smith MA, Perry G, Duffy LK. Melatonin reduces interleukin secretion in amyloid-beta stressed mouse brain slices. Chem Biol Interact 2001;134(1):101-107.

99. Esposito E, Iacono A, Muia C, Crisafulli C et al. Signal transduction pathways involved in protective effects of melatonin in C6 glioma ce1ls. J Pineal Res 2008;44(1):78-87.

100. Esposito E, Cuzzocrea S. Antiinflammatory activity of melatonin in central nervous system. Curr Neuropharmacol 2010;8(3):228-242.

101. Negi G, Kumar A, Sharma SS. Melatonin modulates neuroinflammation and oxidative stress in experimental diabetic neuropathy: effects on NF-kappaB and Nrf2 cascades. J Pineal Res 2011;50(2):124-131.

102. Korkmaz A, Rosales-Corral S, Reiter RJ. Gene regulation by melatonin linked to epigenetic phenomena. Gene 2012;503(1):1-11.

Artículo sin conflicto de intereses 